\title{
The narration "instinct": signalling behaviour, communication, and the selective value of storytelling
}

\author{
Volker Heeschen
}

\section{Introduction: Human ethology, signalling behaviour, and narration}

The title of my essay echoes the title of Steven Pinker's well-known book. Pinker's "language instinct", however, alludes to language structures, whereas my title refers to language functions. Indeed, I will ignore language structures in this essay and concentrate instead on a single language function: the aesthetic use of speech. I will argue that this particular function correlates with - or perhaps even results from - the conditions underlying verbal behaviour in small societies. This claim is somewhat unconventional. For though books on so-called primitive cultures analyse artefacts like cave paintings and carvings, the verbal arts have largely been neglected. They are supposedly too culture-specific. Moreover, questions of evolution and origin were long considered passé in ethnology and linguistic anthropology. The last several years, however, have witnessed a burgeoning of interest in the biological origins of art, morals, virtue, and beauty (on biology and art see Aiken 1998, Cooke and Turner 1999, Dissanayake 1995, Eibl 1995, and Richter 1999; on the origins of moral behaviour see Ridley 1996 and de Waal 1996). In a similar vein, this essay combines research on present-day verbal art, the evolution of verbal behaviour, and the relationships between speakers' aesthetic repertoire and the behaviours enabled and enhanced by language. It proposes that distancing devices coevolved with the artistic uses of language. In other words, what Roman Jakobson (1960) dubbed the "poetic function" is not only responsible for language's aesthetic repertoire. It is also one of the driving forces behind language evolution.

Speech is doubtless a new type of behaviour compared with other forms of communication. The ethnography of communication and linguistic anthropology investigate the functions of speech across different cultures. Yet there is no sub-discipline devoted to studying the evolution of speech. This is partly because speech (unlike, say, tool use) leaves no traces. We can only study present-day communication systems and verbal behaviour. Moreover, ethologists have tended to treat human beings like speechless animals (see Washburn 1978: 414). Yet "etholinguistics" (Eibl-Eibesfeldt 1997: 


\section{Volker Heeschen}

744) and "linguistic ethology" (Sager 1995) can only be pursued succesfully by ethologists (like Irenäus Eibl-Eibesfeldt) who venture into ethnology and by linguists (like Sven Sager) whose research encompasses the nonverbal behaviour of humans and the communicative behaviour of nonhuman primates. Extending the research agenda in this fashion would lead ethologists to topics like the innateness of behaviour, functional equivalences between speech and phylogenetically older communication systems, and the selective value of speech as a behaviour. In other words, it would lead them to topics that have already been studied extensively by psycholinguists, philosophers, and anthropologists.

If the ontogenesis of language acquisition has innate aspects, the urge to know the names of things is one of them. Both children and adults experience a feeling of gratification when they learn the name of an unfamiliar object or person. It is as if the mere act of naming enhances cognition. We also feel an urge to break silences during encounters. Human ethology has systematised these observations and proposed that language helps harmonise social life by establishing distance to emotions, by ritualising aggression, and by mitigating conflicts (Eibl-Eibesfeldt 1997: 744). It has also located similarities between humans and other primates: "The grooming behaviour of nonhuman primates participates in networks of social exchange that share many properties with networks of material object exchange in man" (Reynolds 1981: 199).

Speech is related homologously or analogously to older communication systems. Yet it is also part of the cultural complex that includes the use of symbols in initiations, architecture, painting, carving, and object exchanges. What is the relationship between phylogenetically older communicative behaviour patterns and subsequent symbolic patterns? We have known since Charles Darwin's 1872 The Expression of Emotions in Man and Animals that humans, like other primates, express emotions physiognomically and gesturally. We also know that nonhuman primates are able to think without using names, to solve problems, and to learn by insight. Nonhuman primates regulate group behaviour via nonverbal signals (see Hauser 1996, Lock and Peters 1996, and Hurford et al. 1998). Humans, by contrast, no longer use only nonverbal signals to regulate behaviour or deal with objects. Instead, humans substitute verbal signs for nonverbal gestures and movements. Moreover, what was once instinctive behaviour is increasingly subject to learning and insight. More than half a century ago Konrad Lorenz (1935) observed that what our instincts tell us is not always sufficient: the gap must be filled by learning. Symbolic behaviour has apparently been decoupled from instinct. 
The task of human ethology (and of linguistic anthropology) is twofold. First, it must articulate the path that leads from a code that regulates behaviour to a code that maps environmental and social data. Second, it must articulate a path that leads from thought-without-names to verbal behaviour. In this essay I will not address the common evolutionary phenomena that older functions are accomplished by new morphological means (like behaviour regulation via verbal codes) and that new functions utilise old morphological structures (like language employing the structures of vocal signalling behaviour). Instead, I will concentrate on new functions: representation, narration, and language's inherent aesthetic repertoire.

Biologists like Harry Jerison (1976) and Terry Deacon (1998), who study the coevolution of brain size and symbolic behaviour, have only recently begun to follow in the footsteps of philosophers, linguists, and psycholinguists who have long stressed language's representational function, its distance from emotional states, and its independence from the time and place of the objects it refers to. Jerison asserts that speech and language developed as instruments to "map" new biotopes and to optimally exploit new or plentiful resources (Jerison 1976: 101). According to this view, language is primarily a system for referring to space (and to movement within this space) and to the names of places and living beings. That is, language is principally (and was initially) a system for processing information and for mapping the environment. Jerison maintains that we still need language for designing plans of action (plans for movement in space) and for telling stories. Stories "create mental images in ... listeners that might normally be produced only by the memory of events as recorded and integrated by the sensory and perceptual systems of the brain" (Jerison 1976: 101). For Jerison, communication was an evolutionary side effect

Psycholinguistic, philosophical, and anthropological research on humans' narrative urge concurs with Jerison's distinction between language as a system for mapping reality and speech as a system for communicating. For example, children use language to refer to objects before they learn language's social uses (Freedle and Lewis 1977). Even very young children tell stories, but if you ask them questions or try to interact with them, they frequently remain silent (on the narration-interaction complex, see Heeschen 1988). Adults experience an urge to learn the names of unfamiliar people and objects. Philosophers like Arnold Gehlen (1971: 199) and Hans Blumenfeld (1996: 41) have also emphasised the importance of naming. Learning the names of objects gives them an aura of familiarity, as if we knew them from our own experience. 


\section{Volker Heeschen}

The primary function of myths and nonsacred narratives is to establish order. Merely observing, like Jerison does, that language is necessary to tell stories obscures language's vital functionality. The ethnological literature has amply demonstrated that language's selectional advantages lie in its ability to simulate. Australian aboriginal myths describe travel routes and distant oases, information that could save the lives of parched travellers (Birdsell 1979). Myths report about land settlement and property rights, information that could help settle ownership disputes between rival tribes. And myths record past gift exchanges, information that could tell subsequent generations where to turn to for help in times of need (see Strathern 1971 and Wiessner and Tumu 1998).

Language evolution amounts to a continual levelling out of the differences between language's original mapping function and its subsequent social uses. Language releases humans from primary functional cycles (like eating and copulating) and distances them from their emotions and urges (see Heeschen 1988: 201 and Eibl-Eibesfeldt 1997: 744). Yet language is equally suited to communicating desires, achieving interactional goals, and expressing consent or disapproval by means of digression, misdirection, and play. Hallpike (1985/1986) has demonstrated that although the task repertoires of traditional societies may be comparatively basic, their abundance of symbolic expressions renders such societies highly complex. A single code is not comprehensive enough to handle a wider variety of tasks (begging, for instance, can be accomplished by gestures, glances, and songs). This is why Western societies generalise their codes and use language to reduce ambiguity and to facilitate rapid decision making. They combine language's mapping function and its social uses in ways that differ from those of traditional societies.

Deducing the origins of language from humans' present-day communicative functions and artistic practices is necessarily imprecise. Current communication systems may have developed homologously from early man's nonverbal and verbal behaviour, or they may have emerged analogously in the same environments and under the same functional pressures. Moreover, biological evolution becomes less irrefragable once humans enter the picture. Animals necessarily react in a given way in a given situation. Humans can choose. For instance, humans can respond to aggression by submitting silently, by retaliating immediately, by planning future revenge, by appealing to norms, and by using oratory to appease or ridicule the aggressor. Animal signals must be unequivocal and unmistakable. They result from ritualisation. For Wolfgang Wickler (1967), ritualisation is the process by which intended 
motor activity (and/or motor activity from other functional cycles) assumes the character of a signal. For example, animals bare their teeth when they are about to bite another animal. Ritualisation is the process by which teeth baring itself becomes a minatory signal. Repetition, simplification, and overemphasis "semanticise" signals and render them unequivocal. But unlike signals, language is decoupled from functional cycles, is inherently equivocal, and only achieves full functionality - and acquires adaptive value-when it simulates a reality outside the interactional context. Human language does not guarantee reliability (Zahavi and Zahavi 1998: 371-373). No human society can forego reliable nonverbal signals. And verbal signals only become reliable when they are ritualised and reintroduced into functional cycles (like greeting formulas) or when they become clichés (see Eibl-Eibesfeldt 1988). Ritualisation results in culturally specific social uses of language and speech. The unreliability of language- an unusual feature in the evolution of communication - invites us to search for its adaptive value. Yet new codes have not completely replaced the older systems of signalling behaviour (see Hondrich 1999). In internet chat rooms, icons reintroduce nonverbal signalling behaviour. Visitors enter and exit chat rooms using formulas that are as ritualised as those of stone-age villages.

\section{Speech characteristics in small communities}

A community's size and population density likely determine the speech characteristics of its members. There are no reliable figures for prehistoric population density. Most estimates are based on existing hunter-and-gatherer societies. Figures vary from 0.03 (Herbig 1986: 81) to 0.3 inhabitants per square kilometre prior to the agricultural age. At 0.1 inhabitant per square kilometre, present-day France would have a total population of 55,000 people (Herbig 1986: 81).' After 5,000 BC and during the Bronze Age, population density may have reached 3 or even 17 inhabitants per square kilometre (see Probst 1991 and 1996, L. and F. Cavalli-Sforza 1994, Renfrew 2000, and Birg 1993).

Prehistoric bands likely comprised around 25 individuals, tribes somewhere between 175 and 475 . A band of 25 people had a 50 percent chance of surviving for 177 years. They would have needed to cooperate and establish marriage relationships with other bands. Worldwide, the population of farming villages usually does not exceed 150 (see Dunbar 1996: 92-96). According to Forge (1972), 450 is the maximum size of an egalitarian com- 


\section{Volker Heeschen}

munity; above 450, such communities become subject to fission. Social control becomes problematic in communities that exceed this number. In small communities, a softly spoken utterance is sufficient to reprimand an offender, whereas in larger communities such reprimands provoke arguments and protest. Robin Dunbar (1996) contends that the size of the neocortex correlates with the size of primate groups. That is, the larger the group, the more Macchiavellian intelligence is required to deal with the demands of social life. The optimal and maximum size of human primate communities is 150 .

How do the figures on group size compare with figures on the speakers of single languages? The 750 Papuan languages and the more than 250 Austronesian languages of New Guinea are spoken in an area that measures about 900,000 square kilometres. The average size of a language area is thus about 900 square kilometres - an area that can be traversed on foot (the inhabitable areas are actually somewhat smaller owing to swampland and mountains). This means that each language has an average of 3,000 speakers. Assuming that each language area comprises seven to ten hamlets (as in the valleys of the eastern mountains of West Papua) results in speech communities of 300 to 450 speakers (Heeschen 1992; see also Foley 1986 and Sankoff 1977). Australia and some areas of Africa display similar ratios between population size and language diversity. But considering that some languages in New Guinea are spoken by more than 50,000 people, that exogamous marriage rules and trading partnerships link speakers of different dialects or languages, and that endemic warfare separates speakers of the same language, the average number of speakers per language approaches 1,500 - the presumed size of tribes. Hence, fairly small groups and multilingualism seem to be part of the human condition. The task of this essay is to correlate the small size of ancient and present-day traditional communities and the number of speakers per language with the functions of speech, with verbal and nonverbal behaviour, and with communicative genres.

The vast number of proper names for places, mountains, rivers, ecological niches, paths, borders, and settlements serve to map perceived space and the world beyond one's own experience. Myths and songs are sometimes nothing more than lists of names. Moreover, humans are highly oriented towards space. Myths are frequently accounts of wanderings and tales of territorial occupation. Up to half the vocabulary in conversations and texts implies a spatial reference. More than half of the nouns in languages of mountain-dwelling Papuans refer to plants and animals, all of which can be located. To define things is to delimit their location. Indeed, people enjoy 
naming and describing imaginary spaces; giving accounts of origins and descents; and discovering kin relationships. Speech is the continual projection of a common social and spiritual territory. It replaces the territoriality of the animal kingdom. It also assigns significance to current events by connecting them to the past.

The narration instinct can be thought of as a continuation of children's urge to name people and objects (Lorenz 1973). The naming urge is augmented by the urge to break silences, to give an account of current events, to discover the significance of past events, and to give order to vague plans. Speaking and narrating seem to be means of self-reward and self-gratification.

In Western societies we learn to ask questions, make requests, and provide responses in verbal code. In small non-Western societies people communicate socially via nonverbal behaviour. They transmit knowledge via initiation ceremonies and settle conflicts via gift exchanges. My own research has shown that such societies distinguish more strictly between verbal and nonverbal codes. This accords with observations made in other small societies: "Precontact Fore [a mountain tribe in Papua New Guinea] interaction was based largely on common feeling, personal rapport, and familiarity. Subtle interactive behaviour, not questions and instructions, communicated needs, desires, and interests ..." (Sorenson 1976: 15). And: "In most circumstances [Australian aborigines] do not attempt to constrain others to do their bidding in a direct, overt manner, and nobody is prepared to take orders from others..." (Kendon 1988: 445).

Eipo (in West Papua, where I did research for a number of years; see Heeschen 1998a), Fore, and Australian aborigines use nonverbal codes to transmit information. They do not supplement it with verbal codes, which would draw too much attention to the interaction itself. In small societies, being too blunt is considered aggressive. But all societies transmit desires, needs, and questions verbally, as well. Speakers are confronted with the formidable task of making language - which primarily maps reality - suitable for social uses. They do this by digressing and making detours. They refer to objects outside the immediate interaction, to past events, to absent or never seen objects; they tell stories to appease anger and manage conflicts; and they chat to establish amicable relations prior to reaching decisions. Verbal signs refer to - and simulate - potential actions without initiating them. They divert attention from the immediate interaction, thereby rendering future interactions possible. In other words, verbal signs postpone action.

I stress the importance of digressions' referential and representational character because Malinowski's term "phatic communion" and Dunbar's 


\section{Volker Heeschen}

assertion that speaking is a homology of grooming suggest that the content of utterances is irrelevant. Phatic communion does not convey meaning. It is a type of speech in which "ties of union are created by a mere exchange of words" (Malinowski 1923: 315): "[S]peech is the intimate correlate of [humans' well-known tendency to congregate], for to a natural man, another man's silence is not a reassuring factor, but ... something alarming and dangerous.... The breaking of silence ... is the first act to establish links of fellowship..." (Malinowski 1923: 314). Yet except for very short, highly ritualised formulas like greeting formulas, neither gossip, idle conversation, nor any utterance longer than a noun or verb phrase can do without language's representational function. All utterances rely on naming, on references to time and space - in short, on symbolic behaviour.

Several characteristics of speech favour aesthetic narration. First, speech provides the quintessential means to multiply the steps required to reach a goal. Prolongation makes opposition less dangerous and refusal less likely. For example, when a new kindergartener joins an already existing play group, he or she rarely asks for permission to join the group, although explicit requests are rarely denied (Grammer 1985). The new child establishes contact by observing, imitating, and varying the other children's behaviour. One child wanting to join a play group stood at the window, pointed to a passing aeroplane, and exclaimed: "There's a plane!" The other children stopped playing and looked at the plane. This digression - which refers to an event outside the group - diverts attention, synchronises behaviour, establishes contact, and facilitates admittance into the group. The verbal act is an additional step. This example again illustrates the primacy language's representational function and humans' relatively late acquisition of social language skills.

Second, speakers use misdirection to prolong interaction. Misdirection involves digressing as well as feigning a lack of interest in one's aims, needs, or desires. People talk about things outside their group. They adorn their words with song, oratory, and quotations. In small societies, blunt speech merging verbal and nonverbal behaviour during an interaction - would draw other people's attention to the speaker, filling him or her with shame. Such societies use formalised and ritualised forms of speech like songs and fairy tales to deal with important matters. Blunt words are considered offensive and invite resistance. In small societies, openly calling another person a thief or a miser can have only two outcomes. The accused either strikes his accuser or flees the village in order to avoid censorious glances and gossip. This is why everyday speech in small societies is filled with allusions, tropes, irony, 
veiled speech, wordplay, oratory, and narration. I heard an Eipo woman refer to her own exhaustion by alluding to smoke rising from a nearby mountain and speculating that it came from a fire kindled by a long-suffering woman from the Fa valley exhausted from her daily tasks. Veiled speech and vague allusions are characteristic features of misdirection.

Third, misdirection is augmented by information packaging. Instead of providing concise, precise information, speakers employ prolix utterances in order to maintain secrecy and withhold news. Speech in small communities seems to be less function-oriented and less suited to developing a generalised code for efficient information processing. Speakers are free to comment, tell stories, spin yarns, and play language games. In a society in which everyone knows practically everything there is to know, oratorical skills are used to reawaken interest in familiar topics. Furthermore, new information is too valuable to be revealed prematurely, and small societies handle it "thriftily" (Harrison 1986). Speech is not only a means of communicating, but also of stemming the flow of information and of differentiating oneself from other people. Men set themselves apart from women, initiation groups invent group-specific words, hunters develop their own argot, and speech communities even consciously invent or alter grammatical structures in order to be different from their neighbours and to prevent former allies from understanding them (see Camartin 1992: 39 and 48, Dixon 1997: 13, Heeschen 1998a: 95-102, and Laycock 1982).

Fourth, speech fosters group cohesion and establishes bonds of trust and affection. It helps relieve social tensions (Malinowski 1923, Marshall 1961, and Eibl-Eibesfeldt 1997: 744) and is thought to have replaced grooming as a mechanism for social bonding (Dunbar 1996 and Foley 1997: 67-68). Narrative digressions harmonise social life, and misdirection and information packaging appease conflicting parties. Speech turns objects of primary interest - loved ones, enemies, or strangers - into objects of secondary interest. The speaker no longer sniffs, strikes, or stares, but instead sings a love song, tells about the enemy's origin and descent, or initiates a gift exchange. Speech establishes what the German sociologist Alois Hahn has dubbed Konsensfiktion: fictional consensus (Hondrich 1999: 145). Speech erects a wall of fictions between group members' divergent interests, thereby fostering group cohesion. It can also continue sniffing, striking, and staring by other means. It transposes these actions into a code that is not suited to violence, but that nevertheless invites disagreement.

Digression, misdirection, and fictional consensus make language suited to experimentation - and to lying (Dietzsch 1998: 67-86). Volker Sommer 


\section{Volker Heeschen}

characterises lying as an exercise in mental tickling, mind-reading, and assessing (Sommer 1992: 167). Jean Aitchison describes lying as a valuable skill because it "involves displacement - reference to absent or non-existent events... Furthermore, narrating stories is deeply ingrained in all human cultures: most literature is based on the ability to make nonexistent events plausible" (Aitchison 1996: 21).

\section{Aesthetic form, play, ritualisation}

Speech is essentially an organ for processing information. But once it is decoupled from regulating behaviour and from stimulus-response chains, it can be manipulated playfully and artistically as well as enriched Dissanayake would say: made special - by additional structures, tropes, and enigmatic forms. Such aesthetic features are inherent in our own everyday utterances and in the communicative genres of small societies (see Heeschen 1984; 1998a: 30-35). Speech seems to have a predisposition toward art.

Are these aesthetic forms by-products of language evolution or do they represent additional adaptations? If narration is adaptive behaviour and if telling stories (as Jerison suggests) has selective value, then language and aesthetic forms probably coevolved. If, on the other hand, symbolic behaviour and representation became gradually (and exclusively) assigned to verbal codes, then aesthetic forms probably developed in later phases of language evolution (though what was initially a by-product could have had adaptive value later). There is no easy answer as to why the verbal code was isolated. Older and more recent communication systems - menacing gestures and verbal threats, for example - continue to supplement each other in face-to-face interaction. Yet isolation and detachment do occur. Information is increasingly transmitted on a single channel. Admonitory speeches, songs, veiled accusations, courting, and verbal duelling have gradually become detached from signals that warn of imminent actions. Myths are handed down as narratives and as drama, dance, pantomime, initiation ceremonies, and simple lists of names.

Although the verbal code is universal, the degree of complementarity of the codes is highly culture-specific. Task specificity is crucial. When it comes to comforting, appeasing, teasing, criticising, establishing social bonds, or transmitting accumulated knowledge, speech is a conscious choice from a variety of options. The more or less conscious choice and the isolation of speech from other codes are prerequisites for the appearance of aesthetic forms of communication. These do not function in release-re- 
sponse chains and do not form part of composite signals. Instead, aesthetic forms signal play, peaceful interaction, emotional detachment, experimentation, alternative worlds, and make-believe. They are, in short, vital to human beings. This means that smaller units of speech have selective value to the degree that they tend to develop into narratives.

In addition to play, the driving forces behind language evolution are probably cooperation, conflict management, and prolonged socialisation (Heeschen 1988: 216-222). In traditional societies, cooperation and conflict management require misdirection, veiled speech, and information packaging. You don't say: "Let's plant a new garden." Instead, you chat, gossip, and narrate. The desired cooperation emerges from the reconfirmation of social bonding - not from a direct request. Elaborate speech fosters cooperation by putting participants in the right mood. And speech's ability to establish fictional consensus helps manage conflicts.

Play is an important mediator for elaborate ways of speaking. It is found in children's word games and play acting as well as in secret languages, conscious language alteration, joking, ritualistic insults, and shouting matches of adults (see Goldman 1998, Duranti 1997, Foley 1997, Heeschen 1988 and 1998a; on play and the origins of poetry see Camartin 1992: 184-185). Creativity only emerges during play. Speech consists of a vast number of forms to express a small number needs. Creativity is the positive correlate of what Amotz and Avishag Zahavi call "unreliability" and what Dissanayake refers to as "unpredictability": "In play, novelty and unpredictability are actively sought, whereas in real life we do not usually like uncertainty" (Dissanayake 1995: 43).

In the animal kingdom, play is supposed to prepare young animals for the conflicts and alliances of adulthood. When walking alone in the eastern mountains of West Papua, I was inevitably joined by a child or group of children. One of them would take me by the hand, accompany me for awhile, and perform melodious speeches on the virtues of giving. I was told I should give them pearls, peanuts, fish, and rice and allow them to visit me in my hut. Humans are perpetually threatened by hunger and must be capable of addressing strangers in order to obtain food. Perhaps aesthetic communication is a form of experimental play that prepares us for begging, making friends, and forging alliances in real life.

Prolonged socialisation enables children to address "strangers", that is, members of their society who do not belong to their intimate "security circles" (see Lawrence 1984: 38-60). The Eipo and Yalenang say that children have reached adulthood when they are able to approach a stranger and beg for sweet potatoes. The step outside the security circle, band, or the 


\section{Volker Heeschen}

speech community is the decisive element. This step leads to related but unfamiliar bands, to less familiar members of large villages, and to members of the language community. Within one's own family, band, or speech community, subtle nonverbal behaviour suffices. Outside this circle, speech is required for clarification, and nonverbal behaviour is reduced, controlled, and formalised.

Why do children continue to learn language if one- or two-word sentences and nonverbal behaviour are sufficient for communicating within the security circle? As far as I know, only Jan Gleason has attempted to supply an answer: "[C]hildren have to learn to talk to their fathers and other strangers, and these people are not tuned to them in the warm, sensitive way their mothers are" (Gleason 1973: 293). I interpret Gleason's "father" and "mother" as placeholders for "language community" and "speech community". Ethnological reports on socialisation and childhood show that children are entirely absorbed with establishing social relationships, alliances, and friendships (see Beals 1962: 21-22). Ongoing language learning and honing the capacity to address oneself to strangers are doubtless aspects of prolonged socialisation. Future leaders are usually big talkers. There is a direct relationship between speech, play, and aesthetic forms and the selective value of narration and oratory. Moreover, the size of the speech and language communities correlates with the difference between groups displaying a systematic complementarity of verbal and nonverbal codes and groups in which speech becomes independent. The narration instinct mediates between these two spheres.

But play has rules, creativity must be limited in real life, and speakers choose from many styles, genres, and settings. Creativity, play, and the narration instinct become socially useful via limitation and choice. Unreliable and unpredictable signals are rendered unambiguous. The equivocal signals of play and narration are formalised and ritualised. Ritualisation counteracts language evolution. Verbal material can be reduced to clichés, fragments of older or foreign languages, formulas, and names.

For ethologists, ritualisation has its origins in signalling motions (like the baring of teeth I mentioned above) and acquires semanticity via repetition, exaggeration, and simplification. The new behaviour subsequently becomes an autonomous urge independent of the behaviour in which it originated (Lorenz 1978: 159). Ritualisation serves two functions. It curbs aggression and fosters social bonding (Lorenz 1978: 157). I believe that speech has developed into an independent urge. We cannot remain silent in the presence of others. We feel the urge to say where we come from and where we are 
going. Narration, for example, curbs aggression and fosters bonding by means of digression. Yet what about situations I have labelled Bruchzonen (loosely translated: "danger zones") of social communication: minatory instants when people choose between flight and approach: in encounters with strangers, leave taking, courting, mourning, begging, sharing, admonishing, and managing conflicts? In these danger zones, speaking is no longer or never was - sufficient. Speech is too unpredictable for such situations. Instead, new and unequivocal composite signals gradually developed (or verbal behaviour never achieved complete independence from nonverbal behaviour) to deal with danger zones. For example, eyebrow movement and facial expressions accompany the verbal component of greeting scenes (Eibl-Eibesfeldt 1997: 332 and 633-637).

Like play, many aesthetic forms and communicative genres have constraints. They become composite signals because the symbols of authorship, preferred style, setting, and audience contribute to their semanticity. Below are the major constraints and steps towards semanticity (see Dissanayake 1995: $42-49$ and Heeschen 1984: 407).

First, narration is by definition detached from urges, needs, and desires. Public speeches, sacred narratives, legends, and songs that call for cooperation, cohesion, bonding, or criticism further increase this emotional distance. Nonverbal communication signals are not used. The speaker masks his personality.

Second, linguistic means are reduced. A series of proper names and verbal nouns suffices to create form-meaning relations. Conversely, everyday talk and noncommunicative narratives often employ extremely complicated syntax (see Heeschen 1998 a: 319-359).

Third, the language of communicative genres refers to, and relies on, the meaningfulness of other semiotic systems (the ordering of objects in space, indexicality, and nonverbal signs). A place name, the name of a clan, and the act of keeping one's hands hidden sufficiently indicate an individual, property, and an unwillingness to share. Whereas narratives can make exaggerated use of tropes to a degree that baffles (or awes) the listener, communicative genres must utilise ironic and veiled statements unambiguously.

Fourth, narration tends to use exclusively verbal codes. Some communicative genres re-introduce secondary codes. Myths are sung or use pantomime, public oratory turns into play acting (see Williams 1940 and Salmond 1976). 


\section{Volker Heeschen}

Fifth, narrative and some communicative genres require an audience, whereas poetry, love songs, or speeches criticising prominent people do not. Here, the individual case becomes a general statement.

Sixth, in all genres the participants choose just one of many possible options- namely speech - to address a problem, though the degree of manipulation remains distinctive. Narration pleases, oratory excites, myths indoctrinate, and songs appeal to the emotions.

Seventh, the space is specified. For play acting, performed myths, dancing songs, and public speeches it is the centre of the village. Moreover, performers have narrowly defined roles. Songs can be sung anywhere, though frequently with one condition: the person who is mentioned or criticised in the song should not be present. What is true for play is true for all kinds of communicative performances: "Often special places are set aside for playing: a stadium, a gymnasium, a park, a recreation room, a ring or circle. There are special times, special clothes, a special mood for play think of holidays, festivals, vacations, weekends" (Dissanayake 1995: 43). Wherever people speak, they symbolically mark off an enclosed and consecrated space. Most genres select - and are defined by - places. Narration flourishes around campfires, greeting songs during encounters on the road, and songs wherever someone feels inclined to express his or her emotions (see Heeschen 1984).

Eighth, aesthetic speech creates an atmosphere in which interest is directed away from the original events and toward the pleasure of discovering social meanings in enigmatic forms. This movement away from the object of primary interest and toward the object of secondary interest is precisely what characterises play. Aesthetic speech underscores the metamessage "this is play". This message is inherent in all kinds of speech, especially in every-day talk, gossip, and narration. But it is enhanced in communicative genres by reduction and formalisation so that these genres can serve to curb aggression and foster group cohesion.

Ninth, whereas narration flourishes in the security circles, formalised genres address a wider public, namely "strangers". Experimentation and play make sense within groups of intimate partners. Communicating with "strangers", by contrast, calls for greater prudence, namely: ritualisation.

What role does biology play? The universality of some arts (like bodypainting) and narrative themes (like patricide) provides only inconclusive evidence that art has a biological basis. It is the way humans process information that offers more convincing testimony. Symbols or clichés that evoke 
release-response packages (symbols of territoriality or group cohesion) are universally pleasing. The ability to recognise order and patterns and to decode veiled symbols and messages are prerequisites for the sensory pleasure humans experience in dealing with form-meaning pairings (see Aiken 1998, Boas 1955: 13, Cooke and Turner 1999, and Eibl-Eibesfeldt 1988 and 1997: 899-953): "[H]uman information can be described as ... kalogenetic..., a word coined from the Greek kalos (beauty, goodness, rightness) and genesis (begetting, productive cause, origin, source). The human nervous system has a strong drive to construct affirmative, plausible, coherent, consistent, concise, and predictively powerful models of the world..." (Turner and Pöppel 1988:75). According to Dissanayake, who views art behaviourally (as opposed to, say, historically or sociologically), three indications suggest that art is an evolved behaviour: "The first is ... that it 'feels good', and so people are positively inclined to do it. The second is that people spend a great deal of time and effort doing it. Frivolous pastimes that take energy and time from useful activity are not selected-for, particularly in large numbers of the population, which leads to the third criterion, universality" (Dissanayake 1995: 33). These three characteristics certainly apply to speech, narration, and most communicative genres. Though speech may be a frivolous pastime, I have already alluded to its survival value for children learning to address strangers. Art consists in making "special": embellishing, exaggerating, patterning, juxtaposing, shaping, and transforming (Dissanayake 1995: 38-83). Begging can also be made special by means of patterning, juxtaposing, and repetition. I believe that making "special" and digressions have survival value in societies where people must constantly step outside the security circle and address strangers. The good feeling engendered by speech results from the features it shares with play and ritualisation. These make it an autonomous urge (see Lorenz 1978: 159). Though Dissanayake admits that making "special" also has survival value in the context of "scenario-building", she mainly refers to activities associated with behaviour in the danger zones of human societies: "objects and activities that [were made special] were parts of ceremonies having to do with important transitions, such as birth, puberty, marriage, and death; finding food, securing abundance, ensuring fertility of women and of the earth; curing the sick; going to war or resolving conflict; and so forth" (Dissanayake 1995: 61).

Stories give humans access to alternative worlds, to worlds beyond the horizon, and to behavioural models not sanctioned by familiar rules of sociability and knowledge structures. In traditional societies, borders and horizons are highly significant. The space belonging to an individual or a 


\section{Volker Heeschen}

community is surrounded by symbols. Transgressing or transposing these borders is a symbolic act. Storytellers know how to transcend the ordinary. They know that beyond the horizon things might be radically different. Men might be women. And women might be men. Or, more fantastically, women might own pigs, educate the children, and be the guardians of festivals, manhood, fertility, and thunderstorms. Stories systematically explore alternative worlds (see Eibl 1995: 16).

Creating alternative worlds and making them special via aesthetic forms and structures are biologically endowed needs or predispositions. All genres of poetry and narration depict individuals at work in order to make something special and in order to express personal emotions and opinions. Individuals suffer, individuals ruminate on unsolved problems, and individual narrators rapidly change and mix themes, styles, and genres (see Heeschen 1984 and 1998b; on genres see Luckmann 1988 and Foley 1997: 359-360). Papuan societies are highly individualistic. It seems inconceivable that members of these societies do not construct images of personhood and personal identity. Songs in New Guinea can be highly individualistic (see Feld 1982, Harrison 1986, Heeschen 1984, Strathern 1974, and Finnegan and Orbell 1985). However, like small societies' thrifty approach to new (and thus: valuable) information, images of personhood and personal identity must be handled carefully, toned down, and masked. Stressing the speaker's autonomy would be intolerable in the constant face-to-face communication typical of small communities. Numerous stylistic devices in every-day talk, songs, and speeches serve to mask the speaker's personality, and agenthood.

Speech is a powerful tool for evoking alternative worlds. It is a way to say what should not be said and to enter social spheres beyond one's own security circle. Narration, ritualisation, self-expression, playful speech, and talking to strangers are all, I believe, part of humans' evolutionary history.

\section{Conclusion: narration, hidden information, and veiled communication}

Over the course of evolution, language's mapping and construction-of-reality function has continuously been rendered suitable for social uses. Having become independent of other codes, the verbal code forms composite signals via ritualisation in the danger zones of social life. Misdirection, veiled speech, information packaging, and making "special" (that is, creating aesthetic forms) are correlates of unburdening, distancing, digression, and the unreliability of speech as communication. These capabilities and func- 
tions simultaneously enable speakers to narrate and to create alternative worlds. They are learned during play and enable speakers to act prudently before a wider public and to use ritualised and formalised genres when approaching strangers. Nevertheless, narration and subtle nonverbal behaviour predominate communication in the security circles. The general aim of speech is to create fictional consensus. Finally, there is the individual's urge to express himself or herself, an urge that is less well understood.

In small societies, self, ego, agenthood, and personal interests can only be expressed by aesthetic forms. Here, speech is less burdened with social functions, appeals, criticism, and self-expression must be masked using artistic means. Aesthetic forms of communication thrive in small societies because of the general rule of misdirection and the restrictions that apply to blunt language.

Ritualisation has presumably transformed humans' urge to break silences, to name objects, places, and persons, to recount their own origin and descent, to create playful identities, and to tell stories. It refers to the biology of behaviour and to universal dispositions. Orality and literacy, genres, styles, narration, drama, and poetry are all subjects of extensive research. Yet we rarely ask ourselves why human beings narrate and why they trouble themselves about secrecy, style, making things "special", and creating beautiful things that are neither true nor easily understood. If the aesthetic function is assumed to be at work in all utterances - that is, in both every-day talk and elaborate communicative genres - one might even expect speech and particular language structures to coevolve with humans' drive to tell stories, to keep secrets, and to artistically narrate alternative and nonsensical worlds. In my opinion, the narration instinct is ready to be taken seriously as a concept.

Early human societies were small and isolated. Knowledge was required to arrange meetings at certain times and places. Peaceful cooperation was an urgent necessity for bands of up to 25 individuals. Establishing marriage relationships and trading partnerships was vital in speech communities fewer than 450 people and in language communities fewer than 1,500 members. Means to approach strangers were needed. These factors suggest that mapping the real world, creating alternative worlds, making things "special", making detours, specifying the tasks of distinct codes, and learning the oratory required for communicating with strangers all had survival value. And it is at least possible that there was a correlation between narration and the selective values of misdirection, aesthetic forms, and reality simulation. Distinguishing between speech and signalling behaviour is an important part of the search for the diversity of language origins. 


\section{Volker Heeschen}

\section{Note}

1. Herbig quotes H. M. Wobst, "Boundary conditions for palaeolithic social systems: a simulation approach", American Antiquity 39.2: 147-148. See also Beaken 1996: 125-126, where the relationship between language, exogamous marriage rules, and long distance exchange is mentioned. Though settlement and spreading of homo sapiens, cultures and languages is now widely taken up by geneticists, linguists, and archaeologists, I have not found new figures and calculations, a remark that is also valid for population density. Thematisation of size, density, and language communities is practically nonexistant. 